\title{
Local tuning of photonic crystal nanocavity modes by laser-assisted oxidation
}

\author{
H. S. Lee, ${ }^{1, a)}$ S. Kiravittaya, ${ }^{1}$ S. Kumar, ${ }^{1}$ J. D. Plumhof, ${ }^{1}$ L. Balet,${ }^{2}$ L. H. Li, ${ }^{2}$ M. Francardi, ${ }^{3}$ \\ A. Gerardino, ${ }^{3}$ A. Fiore, ${ }^{4}$ A. Rastelli, ${ }^{1, a)}$ and O. G. Schmidt ${ }^{1}$ \\ ${ }^{1}$ Institute for Integrative Nanosciences, IFW Dresden, Helmholtzstr. 20, D-01069 Dresden, Germany \\ ${ }^{2}$ EPFL Institute of Photonics and Quantum Electronics, Station 3, CH-1015 Lausanne, Switzerland \\ ${ }^{3}$ Institute of Photonics and Nanotechnology, CNR, via del Cineto Romano 42, 00156 Roma, Italy \\ ${ }^{4}$ COBRA Research Institute, Eindhoven University of Technology, $5600 \mathrm{MB}$ Eindhoven, The Netherlands
}

(Received 22 September 2009; accepted 20 October 2009; published online 11 November 2009)

\begin{abstract}
The authors demonstrate a simple method to achieve local tuning of optical modes in GaAs photonic crystal nanocavities by continuous wave laser-assisted oxidation in air atmosphere. By irradiation with a focused laser beam at power levels of a few tens of milliwatts, photonic crystal nanocavity modes shift to shorter wavelengths by up to $2.5 \mathrm{~nm}$. The mode shifts can be controlled either by varying the laser power or by iterating laser-assisted oxidation steps and are well explained by finite-element-method and finite-difference time-domain simulations. This method provides a simple route to achieve fine spectral tuning of individual nanocavities for photonic devices. (C) 2009 American Institute of Physics. [doi:10.1063/1.3262961]
\end{abstract}

Photonic crystal $(\mathrm{PhC})$ nanocavities are attracting much interest for their potential application in advanced optical devices such as switches, ${ }^{1}$ filters, ${ }^{2}$ multiplexers, ${ }^{3}$ lowthreshold lasers, ${ }^{4}$ and cavity quantum electrodynamics. ${ }^{5}$ For such applications, it is necessary to control and tune the resonant wavelength of the $\mathrm{PhC}$ nanocavity modes. The $\mathrm{PhC}$ nanocavity resonances can be tuned by adjusting the $\mathrm{PhC}$ lattice and defect geometries (see, e.g., Ref. 6). However, the exact spectral position of the modes cannot be predicted with the accuracy required for some applications, because the nanocavity resonances are highly sensitive to fabrication parameters. Therefore, postprocessing tuning techniques able to compensate for fabrication imperfections are particularly demanded. Various postprocessing tuning techniques have been demonstrated, such as differential thermal tuning, ${ }^{7,8}$ wet chemical digital etching, ${ }^{9}$ atomic layer deposition, ${ }^{10}$ atomic force microscope nano-oxidation, ${ }^{11}$ liquid crystal infiltration, ${ }^{12}$ and photodarkening of a chalcogenide glass placed on top of the microcavity. ${ }^{13}$ Most of these techniques either modify the properties of the whole sample, which prevents the local tuning of a single nanocavity, or need extra materials and processing tools.

In this letter, we describe a simple method to achieve local tuning of PhC nanocavity modes. It is based on local oxidation produced by continuous wave laser irradiation of single nanocavities in air atmosphere. The modes can be smoothly blueshifted either by varying the laser power or by iterating the laser-assisted oxidation steps. The observed behavior, which is attributed to oxide growth promoted by local heating, is well explained by simulations based on finiteelement-method (FEM) and finite-difference time-domain (FDTD) method.

The investigated two-dimensional (2D) $\mathrm{PhC}$ nanocavities incorporate quantum dots (QDs) acting as broad-band light source and are fabricated on a suspended membrane. The sample, grown by molecular beam epitaxy (MBE), consists of a 320-nm-thick GaAs membrane on top of a 1500-

\footnotetext{
${ }^{\text {a) }}$ Authors to whom correspondence should be addressed. Electronic addresses: h.s.lee@ifw-dresden.de and a.rastelli@ifw-dresden.de.
}

nm-thick $\mathrm{Al}_{0.7} \mathrm{Ga}_{0.3} \mathrm{As}$ sacrificial layer. Three layers of high density InAs QDs with ground-state room temperature (RT) emission at $1.3 \mu \mathrm{m}$ are grown by MBE at the center of the GaAs membrane. The fabrication process consists of patterning of a 150-nm-thick $\mathrm{SiO}_{2}$ mask by electron beam lithography and $\mathrm{CHF}_{3}$ plasma etching and then transfer on the GaAs layer by $\mathrm{SiCl}_{4} / \mathrm{O}_{2} / \mathrm{Ar}$ reactive ion etching. The sacrificial layer is then selectively etched in a diluted HF solution to locally release the GaAs membrane. The investigated $\mathrm{PhC}$ nanocavity consists of triangular lattice $\mathrm{PhC}$ mirrors (filling fraction $35 \%$, lattice constant $311 \mathrm{~nm}$ ) with modified L3 geometry. (For details see Ref. 6). The PhC nanocavities are investigated and laser-processed in a standard microphotoluminescence $(\mu$-PL) setup at RT and in air atmosphere. We use the same laser beam both for characterization (at low power and with a spot size of $\sim 1.5 \mu \mathrm{m}$ ) and for processing (at high power and by defocusing the laser beam to a spot size of $\sim 8 \mu \mathrm{m}$ ). For laser-assisted oxidation, the $\mathrm{PhC}$ nanocavities are irradiated at different laser powers $P$ and by iterating $n$ times the laser-assisted oxidation step with duration $\Delta t=20 \mathrm{~s}$. The excitation source is a frequency doubled continuous wave $\mathrm{Nd}: \mathrm{YVO}_{4}$ laser (wavelength of $532 \mathrm{~nm}$ ) focused onto the sample by means of a $50 \times$ microscope objective of numerical aperture 0.42 . The luminescence is collected through the same objective, dispersed by a spectrometer with $500 \mathrm{~mm}$ focal length, and detected by a liquidnitrogen-cooled InGaAs array detector.

Figure 1(a) shows a RT $\mu$-PL spectrum of an L3 GaAs nanocavity with embedded InAs QDs prior to laser processing. Three optical modes located at 1168, 1217, and $1270 \mathrm{~nm}$ are labeled as M1, M2, and M3, respectively. The result of laser processing [see schematic in the inset of Fig. 1(a)], is shown in Fig. 1(b), where the color-coded $\mu$-PL intensity for the three considered modes is displayed as a function of wavelength and number of laser oxidation steps $n$ at $P$ $=50 \mathrm{~mW}$. The blueshift of modes after laser irradiation is attributed to an oxide layer which forms upon local heating in air atmosphere. Since the oxide has a smaller refractive index than the GaAs slabs, the PhC nanocavity modes shift to shorter wavelengths. Figure 1(c) shows the shifts of M3 

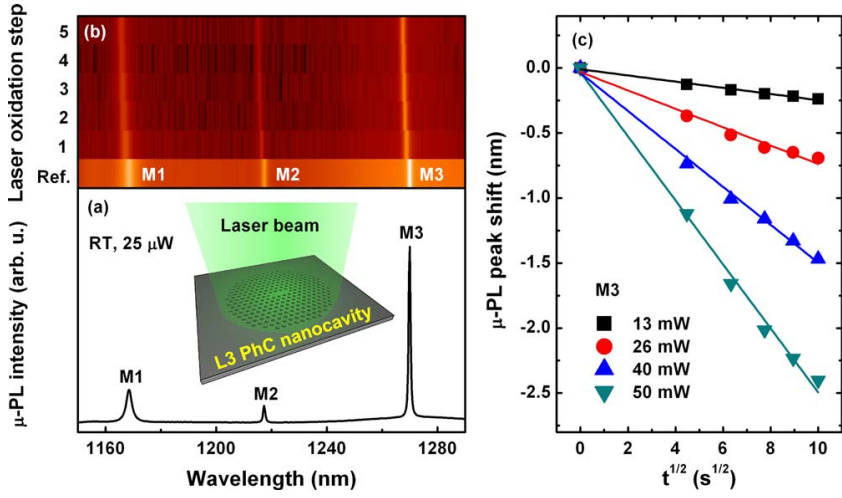

FIG. 1. (Color online) (a) Room temperature $\mu$-PL spectrum of an L3 GaAs nanocavity with embedded InAs QDs. The inset schematically shows the method for local nanocavity tuning by laser-assisted oxidation. (b) Colorcoded $\mu$-PL intensity for three modes (M1, M2, and M3) of the L3 nanocavity as a function of number of laser oxidation steps $n$ at a laser power $P=50 \mathrm{~mW}$. (c) $\mu$-PL peak shifts for M3 modes of different L3 nanocavities as a function of the square root of the oxidation time at the indicated laser powers.

obtained by Lorentzian fits of the $\mu$-PL peaks as a function of the square root of the oxidation time at laser powers $P$ of 13, 26, 40, and $50 \mathrm{~mW}$. The progressive mode shifts with irradiation time $t=n \Delta t$ are attributed to an increase in the oxide thickness $d_{o x}$. By assuming that oxide growth follows the same trend reported for thermal oxidation, ${ }^{14,15}$ we expect that $d_{o x} \approx \sqrt{D_{0}} e^{-E_{a} / 2 k_{B} T} \sqrt{t}$, where $D_{0}, k_{B}, T$, and $E_{a}$ are a constant related to oxygen diffusion coefficient, the Boltzmann constant, the absolute temperature, and the activation energy of oxygen diffusion, respectively. During oxide growth, an amount $\Delta d_{\mathrm{GaAs}}$ of GaAs at the interface between GaAs and oxide is consumed, so that the slab thickness decreases by an amount $2 \Delta d_{\mathrm{GaAs}}$ and the hole radii increase by $\Delta d_{\mathrm{GaAs}}$. We assume $\Delta d_{\mathrm{GaAs}}=c_{o x} d_{o x}$ with $c_{o x}$ being a proportionality constant less than unity. Our FDTD simulations suggest that the wavelength $\lambda_{M i}$ of the mode $\mathrm{Mi}(\mathrm{i}=1,2,3)$ shifts linearly with $\Delta d_{\mathrm{GaAs}}$ with a magnitude of the slope $k_{M i}$ which depends on the mode, $\lambda_{M i}=-k_{M i} \Delta d_{\mathrm{GaAs}}$. These basic considerations lead us to conclude that the wavelength shift $\Delta \lambda_{M i}$ is approximately given by

$$
\Delta \lambda_{M i}=-k_{M i} c_{o x} \sqrt{D_{0}} e^{-\frac{E_{a}}{2 k_{B} T}} \sqrt{t}
$$

From this simple relation we expect that the mode shifts depend on irradiation time according to $\Delta \lambda_{M i} \propto-\sqrt{t}$, in good agreement with the experiment. Figure 1(c) shows in fact that the mode shifts measured at different laser powers $P$ follow straight lines when plotted against $\sqrt{t}$.

In order to understand the dependence on $P$, we first note that an increase of $P$ produces an increase in the temperature of the irradiated region $T$. Since we are not able to experimentally determine the local temperature, we use FEM to estimate the temperature as a function of laser power for the realistic structure and experimental conditions. The temperature profile during laser irradiation is calculated by solving the heat conduction equation, taking into account the temperature dependent thermal conductivity of bulk GaAs. ${ }^{16}$ (We note that the actual thermal conductivity in the $\mathrm{PhC}$ region may be lower than the bulk values due to phonon scattering at the etched interfaces ${ }^{17}$ ). Heat losses due to convection and conduction in air are neglected. The top left inset of Fig. 2(a) shows the calculated temperature profile at the
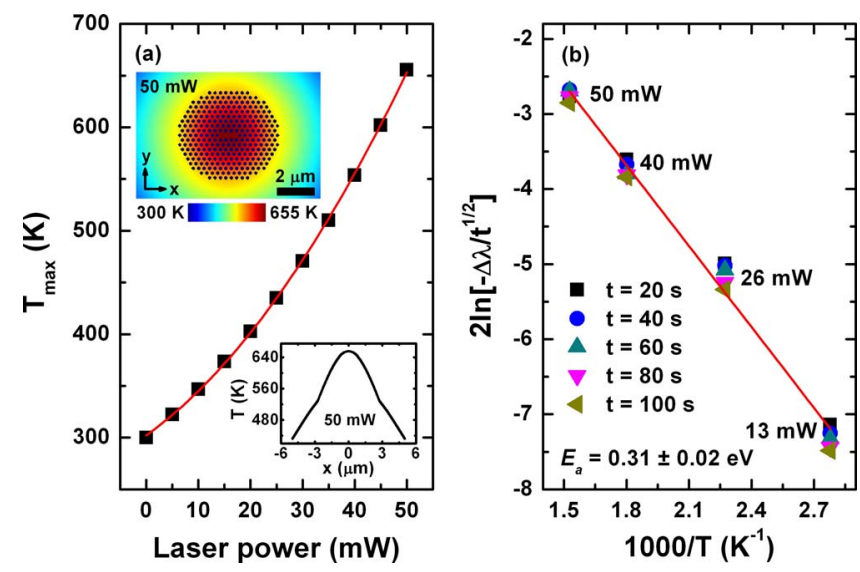

FIG. 2. (Color online) (a) Maximum temperature at the top surface of the L3 nanocavities for laser powers ranging from 0 to $50 \mathrm{~mW}$ calculated by FEM The top left and bottom right insets show the temperature profile at top surface and temperature profile along a $10 \mu \mathrm{m}$ line parallel to the $\mathrm{x}$-axis and $130 \mathrm{~nm}$ away from the center of the L3 nanocavities at a laser power $P=50 \mathrm{~mW}$, respectively. (b) Arrhenius-type plot of the data shown in Fig. 1(c) with temperatures estimated by FEM [see (a)].

top surface of the $\mathrm{PhC}$ nanocavity at a laser power $P$ $=50 \mathrm{~mW}$. As expected, the temperature reaches its maximum value $T_{\max }(655 \mathrm{~K})$ at the center of the laser spot and drops while moving away from it, as seen in the linescan shown in the bottom right inset of Fig. 2(a). From the values of $T_{\max }$ for laser powers ranging from 0 to $50 \mathrm{~mW}$ [Fig. 2(a)] we can now verify the validity of Eq. (1) in describing the mode shifts produced by laser irradiation at different powers. From Eq. (1), we expect $2 \ln (-\Delta \lambda / \sqrt{t}) \propto-1 / T$. This is fully consistent with the experimental results as seen in Fig. 2(b), where the data shown in Fig. 1(c) are displayed in an Arrhenius-type plot. Since the data collected at different powers and different irradiation times follow a straight line, we can estimate the activation energy $E_{a}$ for the oxidation process. From a linear fit of the data shown in Fig. 2(b) we find that $E_{a}=0.31 \pm 0.02 \mathrm{eV}$. (Note that the quoted numerical uncertainty does not take into account the uncertainties in the determination of the actual temperature and the inhomogeneity of temperature profile across the $\mathrm{PhC}$ structure.) For comparison, activation energies for thermal oxidation of GaAs, mostly extracted from experiments performed at higher temperatures, range from 0.25 to $1.1 \mathrm{eV}^{18}$

We now focus on the shifts of the different modes seen in Fig. 1(b) upon laser processing at fixed power. In order to get deeper insight into the shift of these three modes, we performed 2D FDTD simulations with a systematic variation of structural parameters. For the vertical confinement, the effective refractive index theory is used. The dispersion of the GaAs refractive index is taken into account while the refractive index of the oxide is kept constant $(=1.7) .{ }^{19}$ From the simulation, three TE-like mode peaks are observed in the wavelength range $1150-1300 \mathrm{~nm}$. The corresponding electric field intensity distribution profiles are plotted in Figs. 3(a)-3(c). The profiles of different modes overlap differently with the surrounding air holes, so that different shifts can be expected upon oxidation. Figure 3(d) shows the experimentally observed $\mu$-PL peak shifts for these three modes (M1, M2, and M3) and the quality factor $Q$ of the M3 mode as a function of the square root of the oxidation time at a laser power $P=50 \mathrm{~mW}$. The quality factor of the M3 mode decreases by $\sim 23 \%$ with increasing oxidation time at a laser 

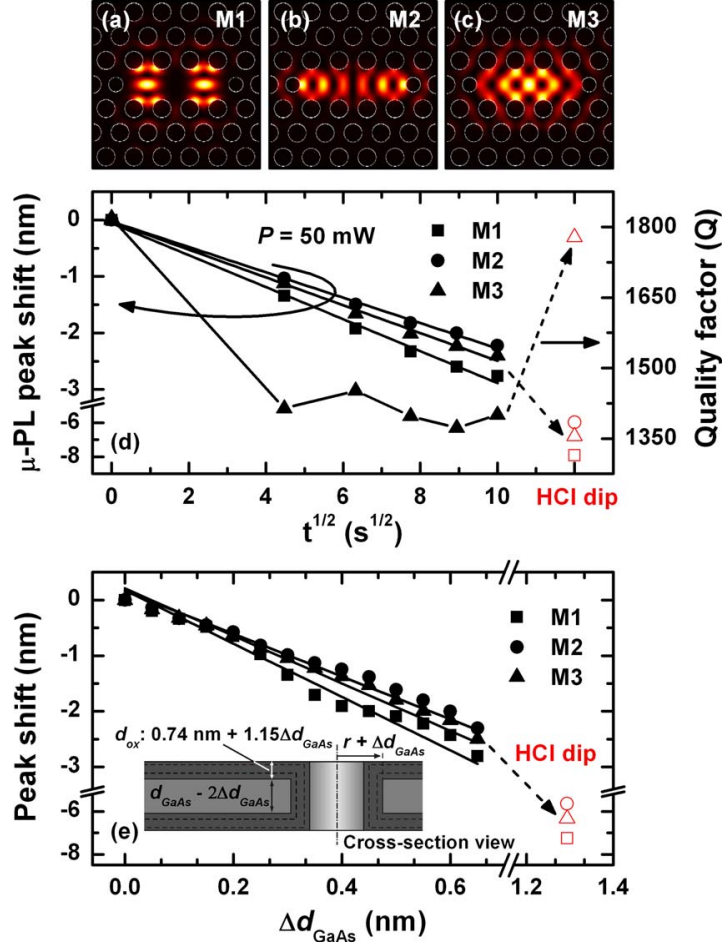

FIG. 3. (Color online) FDTD-calculated electric field intensity profiles for the TE modes (a) M1, (b) M2, and (c) M3 confined in the L3 nanocavity. (d) $\mu$-PL peak shift for the three modes and quality factor for M3 as a function of the square root of the oxidation time at a laser power $P=50 \mathrm{~mW}$. The open symbols indicate the values after a dip in $\mathrm{HCl}$ for $5 \mathrm{~s}$. (e) Simulated peak shifts for the three modes as a function of the change of hole radii $\left(\Delta d_{\mathrm{GaAs}}\right)$ and slab GaAs thickness $\left(2 \Delta d_{\mathrm{GaAs}}\right)$. The peak shift produced by $\mathrm{HCl}$ dip at the final step (open symbols) is calculated by further reducing the GaAs slab thickness, increasing the hole radii and reducing the oxide thickness $\left(d_{o x}\right)$ to the original value $(0.74 \mathrm{~nm})$. The inset shows the crosssectional schematic of the GaAs slab with a hole. Geometry changes by laser oxidation are marked (see text for more detail).

power $P=50 \mathrm{~mW}$, as shown Fig. 3(d). This behavior can be ascribed to the roughness of the oxide layer and possibly to an increase of the density of surface states, with a consequent increase of absorption losses. However, the initial $Q$ values can be recovered after a dip in $\mathrm{HCl}$, which smoothens the surface of the oxide layer [open triangle in Fig. 3(d)]. ${ }^{20}$

Different modes show slightly different shifts, with increasing oxidation time. For example the M1 mode shifts faster than M2 and M3. We simulate the laser oxidation steps by gradually decreasing the GaAs slab thickness by $2 \Delta d_{\mathrm{GaAs}}$ and increasing the hole radii by $\Delta d_{\mathrm{GaAs}}$ while the oxide thickness $d_{o x}$ is increasing from the value of typical native oxide thickness $(0.74 \mathrm{~nm})$ (Ref. 21) with the rate $1.15 \Delta d_{\mathrm{GaAs}}$, which is obtained from the simultaneous fitting of these three modes [see the inset of Fig. 3(e)]. Figure 3(e) shows the wavelength shift for the three modes as a function of the change in GaAs thickness due to the oxidation process. The trends of the peak shifts of the three modes agree well with the experiment, i.e., $k_{M 1}>k_{M 3}>k_{M 2}$ [see Eq. (1)]. Furthermore linear fits of the simulated data give $k_{M 2}=0.80 k_{M 1}$ and $k_{M 3}=0.89 k_{M 1}$, in good agreement with the experimental results obtained by linear fits of the data shown in Fig. 3(d) versus $\sqrt{t}\left(k_{M 2}=0.79 k_{M 1}\right.$ and $\left.k_{M 3}=0.87 k_{M 1}\right)$. In addition, the simulation allows us to quantify the amount of oxide formed in each laser oxidation step or removed during the $\mathrm{HCl}$ dip as shown in Fig. 3(e).
In conclusion, we have investigated the local tuning of $\mathrm{PhC}$ nanocavity modes by laser-assisted oxidation. The $\mathrm{PhC}$ nanocavity modes shift to shorter wavelengths with increasing laser power and by iterating laser oxidation steps, which we attribute to the thermally induced growth of an oxide layer upon local heating. The achieved tuning range $(\sim 2.5 \mathrm{~nm})$ already allows compensating inevitable fluctuations of the cavity resonance over nominally identical devices on a wafer. The method could complement nonlocal and coarser tuning techniques such as digital etching using citric acid, ${ }^{9}$ hydrochloric acid, ${ }^{20}$ or hydrofluoric acid ${ }^{22}$ solutions, which produce wavelength shifts of a few nanometers per cycle. Since the trend of thermal oxidation in silicon is similar to that in $\mathrm{GaAs}^{23}$ and laser-assisted oxidation of $\mathrm{Si}$ has been demonstrated, ${ }^{24}$ we envision the applicability of the method for fine-tuning not only of GaAs- but also of $\mathrm{Si}$ based nanophotonic devices. ${ }^{7}$

The authors gratefully acknowledge A. Ulhaq and M. Benyoucef for their contribution to the measurement and F. Römer, B. Witzigmann, and P. Atkinson for fruitful discussions. This work was supported by the DFG (FOR 730). One of the authors (H.S.L.) was supported by the Korea Research Foundation Grant funded by the Korean Government (Grant No. KRF-2008-357-C00035).

${ }^{1}$ T. Tanabe, M. Notomi, S. Mitsugi, A. Shinya, and E. Kuramochi, Appl. Phys. Lett. 87, 151112 (2005).

${ }^{2}$ B. S. Song, S. Noda, and T. Asano, Science 300, 1537 (2003).

${ }^{3}$ K. H. Hwang and G. H. Song, Opt. Express 13, 1948 (2005).

${ }^{4}$ M. Lončar, T. Yoshie, A. Scherer, P. Gogna, and Y. Qiu, Appl. Phys. Lett. 81, 2680 (2002).

${ }^{5}$ T. Yoshie, A. Scherer, J. Hendrickson, G. Khitrova, H. M. Gibbs, G. Rupper, C. Ell, O. B. Shchekin, and D. G. Deppe, Nature (London) 432, 200 (2004).

${ }^{6}$ M. Francardi, L. Balet, A. Gerardino, C. Monat, C. Zinoni, L. H. Li, B. Alloing, N. Le Thomas, R. Houdré, and A. Fiore, Phys. Status Solidi C 3 , 3693 (2006).

${ }^{7}$ J. Pan, Y. Huo, K. Yamanaka, S. Sandhu, L. Scaccabarozzi, R. Timp, M. L. Povinelli, S. Fan, M. M. Fejer, and J. S. Harris, Appl. Phys. Lett. 92, 103114 (2008).

${ }^{8}$ A. Faraon and J. Vučković, Appl. Phys. Lett. 95, 043102 (2009).

${ }^{9}$ K. Hennessy, A. Badolato, A. Tamboli, P. M. Petroff, E. Hu, M. Atatüre, J. Dreiser, and A. Imamoğlu, Appl. Phys. Lett. 87, 021108 (2005).

${ }^{10}$ X. Yang, C. J. Chen, C. A. Husko, and C. W. Wong, Appl. Phys. Lett. 91, 161114 (2007).

${ }^{11}$ K. Hennessy, C. Högerle, E. Hu, A. Badolato, and A. Imamoğlu, Appl. Phys. Lett. 89, 041118 (2006).

${ }^{12}$ F. Intonti, S. Vignolini, V. Türck, M. Colocci, P. Bettotti, L. Pavesi, S. L. Schweizer, R. Wehrspohn, and D. Wiersma, Appl. Phys. Lett. 89, 211117 (2006).

${ }^{13}$ A. Faraon, D. Englund, D. Bulla, B. Luther-Davies, B. J. Eggleton, N. Stoltz, P. M. Petroff, and J. Vučković, Appl. Phys. Lett. 92, 043123 (2008).

${ }^{14}$ M. Fukuda and K. Takahei, J. Appl. Phys. 57, 129 (1985).

${ }^{15}$ K. Navratil, Czech. J. Phys., Sect. B 18, 266 (1968).

${ }^{16}$ Properties of Gallium Arsenide, EMIS Datareviews Series, 3rd ed., edited by M. R. Brozel and G. E. Stillman (INSPEC, London, 1996).

${ }^{17}$ D. Song and G. Chen, Appl. Phys. Lett. 84, 687 (2004).

${ }^{18}$ A. Rim and R. Beserman, J. Appl. Phys. 74, 897 (1993).

${ }^{19}$ P. A. Barnes and D. P. Schinke, Appl. Phys. Lett. 30, 26 (1977).

${ }^{20}$ T. Sünner, R. Herrmann, A. Löffler, M. Kamp, and A. Forchel, Microelectron. Eng. 84, 1405 (2007).

${ }^{21}$ Y. Mizokawa, O. Komoda, and S. Miyase, Thin Solid Films 156, 127 (1988).

${ }^{22}$ D. Dalacu, S. Frédérick, P. J. Poole, G. C. Aers, and R. L. Williams, Appl. Phys. Lett. 87, 151107 (2005).

${ }^{23}$ P. R. Lefebvre and E. A. Irene, J. Vac. Sci. Technol. B 15, 1173 (1997).

${ }^{24}$ F. Micheli and I. W. Boyd, Appl. Phys. Lett. 51, 1149 (1987). 